TP Periodica Polytechnica Electrical Engineering and Computer Science

59(3), pp. 110-117, 2015

DOI: 10.3311/PPee.8574

Creative Commons Attribution (i)

RESEARCH ARTICLE

\section{Grid Modeling Including Consumption and Distributed PV Generation with Focus on Voltage Profile}

\author{
János Csatár ${ }^{1 *}$, András Dán ${ }^{1}$
}

Received 17 May 2015; accepted after revision 21 August 2015

\begin{abstract}
Distributed generation is growing rapidly and it poses challenges to grid operations, especially on LV network. A set of sample networks are created based on the Hungarian LV distribution network to study the effects of distributed generation. These models represent accurately the overall LV network structure. Thus, conclusions based on them are valid for the Hungarian LV distribution network. Consumer models are also constructed, with focus on random behavior, based on actual measurements. The model networks utilize PV generation - as this gives over 99\% of the DG in Hungary. Simulations are carried out on this model set to find the limits of PV $D G$ in the network by voltage profile, without any smart control. Overhead lines will face voltage problems - even with just a small amount of PV generation, while cable networks can keep a good voltage profile. Two simple regulation methods are also tested.
\end{abstract}

\section{Keywords}

Benchmark network, Distributed power generation, Load modeling, LV network, Network modeling, Voltage control
${ }^{1}$ Department of Electric Power Engineering, Faculty of Electrical and Informatics Engineering, Budapest University of Technology and Economics H-1521 Budapest, P.O.B. 91, Hungary

*Corresponding author, e-mail: csatar.janos@vet.bme.hu

\section{Introduction}

Distributed generation is growing rapidly and it poses challenges to grid operations, especially on LV network. A set of sample networks helps to study the effects of distributed generation on current LV networks.

The first step of the research on LV network, is modeling. More precisely the network topology, generation, and consumption.

Microgrid models that has similar basic characteristics to current networks are also needed. Most of the microgrid studies are based on a very simplified network (e.g. one bus bar - one consumer- few generation units [1]) or based on real network data - although a specific one [2]. A commonly used European benchmark lv network [3] exists to support researches; however, it is also a more simplified case. In these cases, extending the conclusions to other types of network requires further analysis - especially in the case of voltage profiles.

A comprehensive set of benchmark networks has not been found to date. To address this issue, the current paper is analyzing the Hungarian LV distribution network, and a set of sample networks are created. These samples represent accurately the overall Hungarian LV network structure. Therefore, conclusions based on these models are valid for the Hungarian LV distribution network.

Consumption is also part of the current model. The network side characteristics of equipment found in ordinary households is slowly changing. E.g. an appliance consuming power, which is neither frequency nor voltage dependent - while it operates normally. One other problem is the hardly predictable and dynamic nature of the consumption - it has a great variance (spikes and nearly no load) in an LV network. The accurate modeling of a real consumer is a difficult, but essential requirement. While there are some works related to model residential consumption (e.g. $[4,5,6])$, the set of parameters needs special attention and still not guarantee the accurate modelling of load spikes and periods with low loading. These parameters could be determined from sample measurements and need verifying. As a large database of load measurement is available, it is more precise and straightforward to use it directly, instead of parameter tuning. This way, the 
model inherently also incorporates Hungarian household consumer behavior - most importantly the random changes.

Last by not least, the generation. Whereas the consumption is very stochastic - varying from household to household - and cannot be controlled to a large extent, the generation is normally easier to control. However, all of the sources are operating identically along the feeder in the case of PV generation without control. As PV generation gives over $99 \%$ of the distributed LV generation in Hungary, the model network will utilize this type of generation.

There are numerous works related to voltage profiles with increased PV generation (e.g. [7]-[12]), but this article is dealing with the specific behavior of the constructed model networks. Therefore - based on these models - simulations are carried out to find the limits of distributed generation by voltage profile, without any smart control. Two simple regulation methods are also tested to show how even these simple methods improves the acceptable PV penetration on the network.

\section{Network Data Analysis}

Hungarian distribution network operators provided the data, which covers around 5/6-th of the data available on Hungary's LV network - representing all kinds that occur, including rural, suburban, and urban areas. Only the southeastern part of the country is not included in these dataset. However, the LV network structure in the country is very similar in the same type of areas, i.e. urban, suburban, or rural.

The goal was to represent feeder lines and make models for them, so aggregated values were calculated from the primary data by feeder lines. For each feeder line a graph was constructed, from which parameters were derived, consisting, among others, of:

- Overall length of the wires

- Number of leaf nodes

- Maximum leaf distances from the transformer

- Average leaf distance from the transformer

- The above mentioned parameters in electrical distances

- How many connections could be made with neighboring lines in practice

- Ratio of overhead lines vs underground cables

- Number of customers supplied

- Calculated current at the root node

- Consumer per length, current per length

Some basic principles helped to reduce the complexity of the task.

Firstly, too short lines are less interesting (below $20 \mathrm{~m}$ ). These are usually serving only one customer, moreover such short distances are not going to have any voltage profile problems either.

Secondly, extremities do not need to be covered. While extreme cases can cause interesting problems, the goal of this article is to give general tendencies. As such, a few problematic lines are not representing the overall situation on the network. These very extreme cases are known by the DSO-s and will undergo network reconfiguration and reinforcement in the near future. Moreover, the problems identified by the model networks most definitely also exists with these extreme cases.

Thirdly, the goal is to make a set of model networks on which typical problems will appear. Therefore using assumptions towards the worst case (extremities should be avoided) simplification could be made.

IBM SPSS software provided the statistical calculations. Looking at the statistics of different DSO-s reveals that the networks do not differ much. The only exception was Budapest, which is a little different in mainly shorter lines, more cables, and more load. Nevertheless, comparing Budapest to other cities' cable lines shows similar networks.

The aggregated values reduced the amount of data, but it was still too much. Thus principal component analysis was used to find the inner dimensions and dependencies. The chosen set of dimensions covered $95 \%$ of the deviations. The following key parameters could be distinguished:

- The general size of the network (both by means of physical and electrical length)

- Overhead or cable network

- The number of laterals

- The number of connections to other lines

- The load of the line

- The number of consumers on the line

The important thing is that these dimensions (not counting the overhead or cable dimension) show little correlation with each other. This means that, based on the type of network (i.e. cable or overhead), the other dimensions are independent from each other. Further analysis of the data revealed that there are not many possible values in those dimensions. It also helps to concentrate on longer lines and greater loads, as a lightly loaded, short line is most likely to be the last to pose operational challenges.

Half of the network is underground cable with mostly 1 $(75 \%)$ or $2(12 \%)$ leaf nodes (end points of the line not counting the transformer). They characterize urban areas. The overhead lines comprise 1 (35\%), $2(25 \%), 3(15 \%)$ or $4(10 \%)$ leaf nodes. Rural and suburban networks mostly consist of these overhead lines. The rest of the cases range from 3 to 8 in case of the cables and from 5 to 25 in the case of overhead lines.

The next variable is the length of the line, more precisely the point with the maximum distance. Again, the cable and overhead lines are entirely different. Cables range from 0-700 $\mathrm{m}$ and overhead lines range from $0-1500 \mathrm{~m}$. There are not any clear groups here; it is similar to a Weibull distribution. The length can be divided into $30 \mathrm{~m}$ sections; this is the average pole distance in the case of overhead lines. This can be also stipulated 


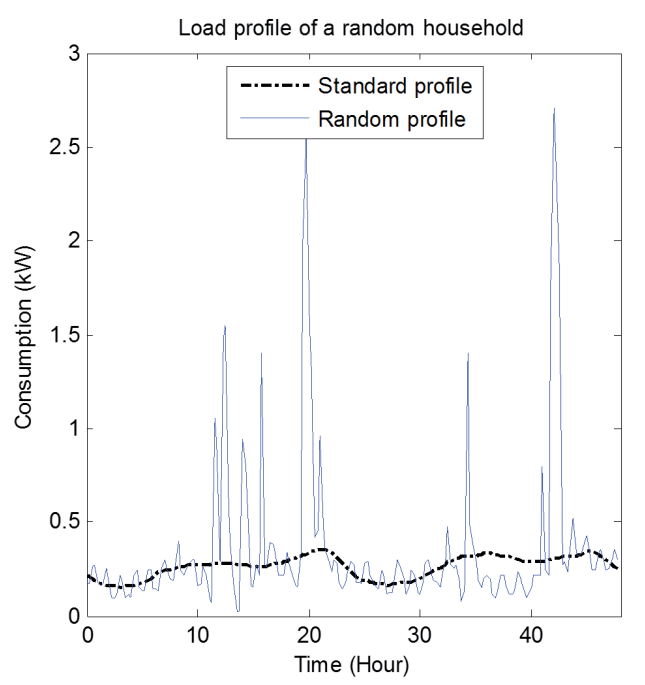

Fig. 1 Two day (Friday - Saturday) comparison of the standard load profile and a random, measured load profile, both have a yearly consumption of $2500 \mathrm{kWh}$.

at the cable lines, because this section length is appropriate for average house distances.

Fortunately, the distance correlates with the maximum distance in the case of laterals. Subsequently, the maximum distance also defines the lengths of laterals and the connection point to the backbone.

The possible interconnection points of the networks are 0-1 for cables and 0-1-2 for overhead lines. These points were placed to different ends of the network, although not used here for calculations.

The types of conductors are $95 \mathrm{~mm}^{2}$ and $50 \mathrm{~mm}^{2}$ aluminum wires in the case of overhead lines, and $150 \mathrm{~mm}^{2}$ and $240 \mathrm{~mm}^{2}$ aluminum in the case of underground cables. The backbones are of the greater diameter, and usually the laterals use the thinner wires.

As for the consumption, $80 \%$ of the overhead lines supplies one consumer at every 10-30 m. The cable lines serve around three times as many consumers per meter as overhead lines.

Generation is scarce in today's Hungarian LV network. There are some separated cases. Although yet uncommon, distributed generation is growing dynamically, doubling the built-in capacity every year. Thus, soon it will become a major concern for DSOs to keep the network in normal operational condition. Especially because only PV generation is growing, where peak production and peak demand occur at different times with residential consumers.

\section{Consumption and Generation}

Usually household consumers give a large portion of load in an LV network. Household consumption is very hard to model precisely, mostly because it changes dynamically to a large extent and the consumer behavior varies from household to household. Still, statistically made standard consumption profiles are readily available for calculations. However, this is

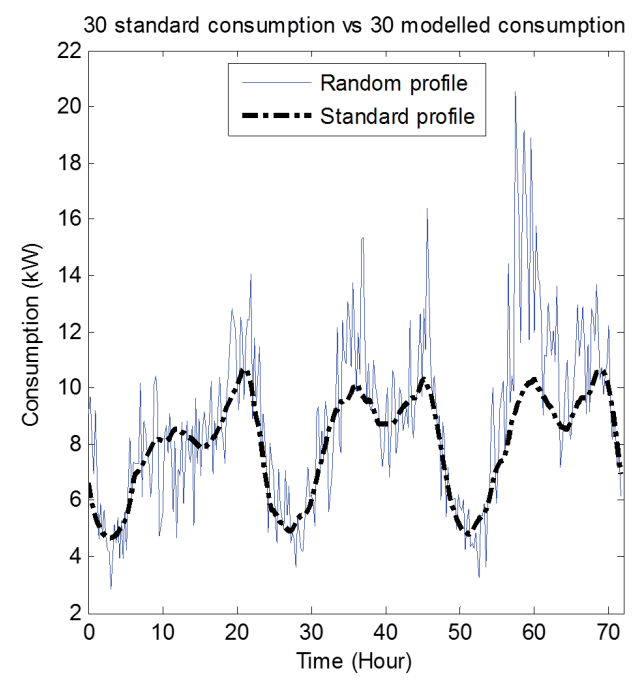

Fig. 2 Three day (Friday - Saturday - Sunday) comparison of the sum of 30 standard load profiles and the sum of 30 random, measured load profiles, both set of profiles have a mean yearly consumption of $2500 \mathrm{kWh}$.

smooth and is good only for a large number of customers clustered together. This standard profile is lacking any fast or large change in consumption. (See Fig. 1)

In the case of single feeders, there are not enough customers to smooth the consumption, even more, they are correlated (e.g. going to work at morning, and coming home at afternoon, using more energy at early night than at noon). This can be clearly seen on Fig. 2. In this example, the consumption of 30 households is calculated using the standard residential household load profile and it is compared to a set of 30 random measured residential profiles - There are large deviations both in the positive and negative range.

A sample year for 1422, metered customers was made from a large database of 15 minutes measurements spanning across years. The typical household consumers show two special characteristics: more energy usage at late afternoon and night, than in the daytime, and more energy usage at weekends than on weekdays. These simple rules sorted out commercial customers and leaved 426 household consumers (from the 1422). These profiles have a mean yearly consumption of $2500 \mathrm{kWh}$ ranging from $800 \mathrm{kWh}$ to $8000 \mathrm{kWh}$. The network has less loading at daytime on weekdays than on weekends, so the calculations used weekday profiles.

Commercial consumers are usually supplied by shorter lines and are not on the same feeder as residential consumers. Since PV generation produces power at daytime and commercial consumers use more energy at this time, problems are less likely to occur in these cases than with residential load. In the latter case, peak load is at late afternoon and night, when there is little or no PV generation, and when the PV generation is at its peak, the network is lightly loaded. Moreover, residential type consumers are dominant on longer lines. Therefore, it is preferable to use residential consumption models to search for problems that arise with growing distributed PV generation. 
For this reason, only residential customers were used for the calculations.

PV generation was estimated using a year's normalized production curve, according to which, a $1 \mathrm{~kW}$ peak system can produce around $1300 \mathrm{kWh}$ of energy a year. If a consumer decides to build PV generation, the system most likely will be able to produce roughly the same amount of energy that the customer normally uses in a year - due to legislative environment.

PV penetration for simulation purposes here represents the chance that a customer is equipped with PV generation. For example with a PV penetration of $20 \%$ around every fifth household will be equipped with PV generation. If a household is picked for generation, its peak power is calculated based upon its yearly energy usage.

During the simulations, every consumer is assigned to a random profile from the previously mentioned 426 cases. The simulation is conducted for a full day, meaning 96 calculations for a day. To increase the possible combinations, every $15 \mathrm{~min}$ utes is used from a different profile and a different day, but in the same time of the day (e.g. every profile's load is from 18:15 when calculating the 18:15 time step). Attention was made to include day types. In a simulation case, all of the used profile measurements are from the same type of the day (i.e. weekday, Saturday or Sunday). Only loads from summer days were used to study generation and a clear sunny day was stipulated.

As only the active power was measured, the reactive power was estimated for each time step for each consumer with a random power factor ranging from 0.86 to 1 (inductive) below 500 $\mathrm{W}$ power usage and from 0.95 to 1 (inductive) for greater loads. These values originate from a series of measurements that involved six households. These measurements were recorded at the PCC (point of common coupling) and has a resolution of 1 minute. For PV generation a power factor of 1 was used.

\section{Model Networks}

There are a total of 11 model topologies of which 2 are cable and 9 are overhead lines:

- The 2 cable models (Fig. 4) have

- 1 and 2 leaf nodes respectively.

- a maximum distance of $450 \mathrm{~m}$

- 9 household per section (30 m)

- $150 \mathrm{~mm}^{2}$ and $240 \mathrm{~mm}^{2}$ aluminum cables

- The overhead models (Fig. 3 and Fig. 5) have

- 1, 2, 3 and 4 leaf nodes respectively.

- 3 household per section $(30 \mathrm{~m})$

- $95 \mathrm{~mm}^{2}$ and $50 \mathrm{~mm}^{2}$ aluminum wires

- Multiple maximum distance configuration:

- $500 \mathrm{~m}$ (1,2 and 3 leaves)

- $750 \mathrm{~m}$ (3 and 4 leaves)

- $990 \mathrm{~m}$ (1,2,3 and 4 leaves $)$
The overhead models have a little different topology depending on their maximum distance. Variations in maximum distance were included for two reasons: Firstly, the basic length dependency of the calculated values. Secondly, to have lines that supply around the same number of customers as the lines with fever laterals. These models are built of $30 \mathrm{~m}$ sections. The endpoints of the sections are also the points of common coupling for consumers. This corresponds well with average pole distance for overhead lines, but is also valid for cable networks because of the house distances. Three household consumers are connected to every PCC with an overhead line, and nine household consumers with a cable line. Most of these consumers are using single-phase connection. The laterals in overhead networks are of smaller cross-section than the backbone.

These parameters were statistically calculated using the network dataset mentioned in Section 2. The goal was to represent the general voltage profile tendencies in the country with a handful of model networks. Therefore, some extreme topologies were neglected. Longer lines and more household were favored.

The overhead line topologies will be referred to by their number of leaf nodes, and the maximum distance.

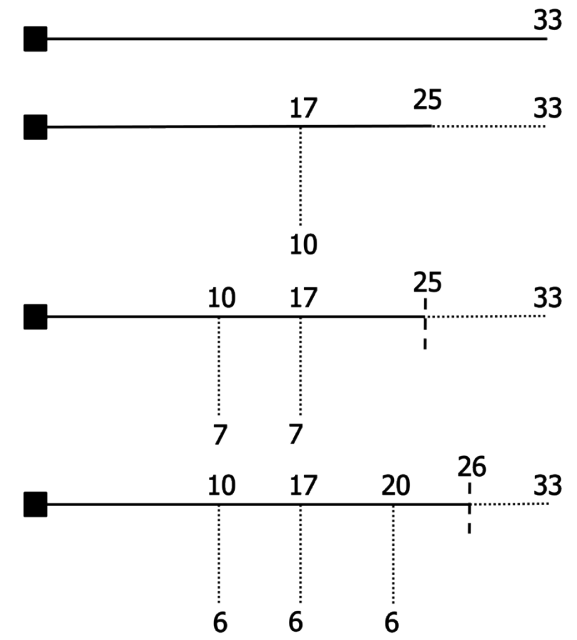

Fig. 3 Model network topologies for overhead lines $(990 \mathrm{~m}$ and $750 \mathrm{~m}$ versions). The numbers show distances in $30 \mathrm{~m}$ sections (average LV pole distance). Continuous and dotted lines represent 95 $\mathrm{mm}^{2}$ and $50 \mathrm{~mm}^{2}$ cross sections respectively. The marked points in the last two cases show the endpoints of the $750 \mathrm{~m}$ versions.

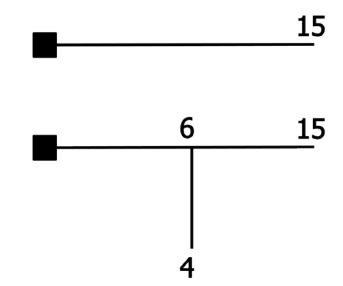

Fig. 4 Model network topologies for cable lines. The numbers show distances in $30 \mathrm{~m}$ sections. 


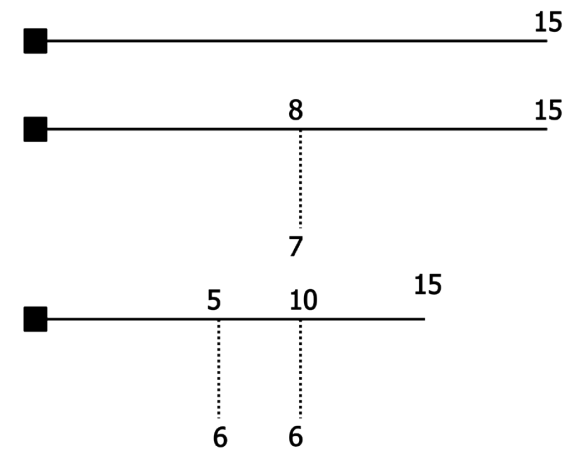

Fig. 5 Model network topologies for overhead lines (500 m versions). The numbers show distances in $30 \mathrm{~m}$ sections (average LV pole distance). Continuous and dotted lines represent $95 \mathrm{~mm}^{2}$ and $50 \mathrm{~mm}^{2}$ cross sections respectively.

\section{Simulation Results without Smart Control}

Voltage profiles were analyzed to determine the allowable PV penetration on the model networks. The simulations were calculated with Matlab utilizing a load-flow, based on forwardbackward sweep method - with BIBC (busbar injection to branch current) and BCBV (branch current to busbar voltages) matrices [13]. Active and reactive power is not voltage dependent. It makes sense as more and more of today's equipment is supplied by power electronics and PV generation is also supplying through power electronics. While a typical household's load is not strictly voltage independent, the error neglecting it is small around the nominal voltage. Furthermore, PV generation is voltage independent and this is more important in this study. One feeder was computed at a time, thus the effects of the transformer were ignored. A sunny summer day with clear sky was used to estimate PV generation.

Only single line models were calculated, since the uncertainty in the distribution of consumers introduces greater variance than the effect of other phases. Nevertheless, it is worth noting, that system asymmetry could introduce greater voltage drop.

The root node (busbar voltage) voltage was set to $240 \mathrm{~V}$, because the MV/LV transformers use a fixed tap setting. At the winter peak load - which is closely followed by the summer night peak load - this source voltage is necessary to maintain voltage everywhere within the limits. This starting voltage is commonly found in real networks. The transformer would further increase this voltage at daytime with high PV penetration even if a fixed tap setting were used.

Every simulation case was run 50 times, to explore the possible situations. The mean value and the $90^{\text {th }}$ percentile voltage for every 15 minutes were calculated at every node. The lower $10^{\text {th }}$ percentile voltage was also calculated and it was found that all of the sample networks supplied even the winter night's peak load. These percentiles leaves out 5-5 cases from the 50 runs - there is a step around the $90^{\text {th }}$ and $10^{\text {th }}$ percentile. This way the typical worst cases could be showed. The voltage drop never exceeded $20 \mathrm{~V}$. Since the root node has $240 \mathrm{~V}$, the network has at least $220 \mathrm{~V}$ everywhere.
The CIGRE residential benchmark system has also been included for comparison purposes. Since it has just a few PCC$\mathrm{s}$, the PV penetration was handled manually, equipping consumers starting from the end of the network, adding new PV generation every $20 \%$.

The nodal voltage sensitivity was also calculated, and it was found that it could be well linearized. It gives more insight into the networks' robustness. With overhead lines- near the leaf nodes (the endpoints of the line) - this meant generally around $6 \mathrm{~V}$ per $3000 \mathrm{VA}$ changes (both in the positive and in the negative range, with both reactive and active power). If overhead cable were used instead of overhead bare wire, the sensitivity to reactive power injection would decrease to around $1 / 8$ th of the original value.

With these sensitivities, even a single PV generation of a few $\mathrm{kW}$ built further from the transformer may introduce problems. Given that power electronics in PV generation have general overvoltage protection it will not generate power at times with high solar irradiation, instead it would simply switch on and off continuously. Of course, this depends on the amount of solar irradiation on the PV panels in that moment. Figure 6 shows a node's sensitivity that is at the end of the line in a long straight line configuration. It can be observed that the reactive power injection causes around the same voltage change throughout the day, but the active power is more dependent on the network loading. This correlates to the fact that PV generation and higher load values have a power factor close to 1. Due to this, using an overhead cable or bare wire configuration does not change the general behavior of the system. This is true while the reactive power flow is not significant.

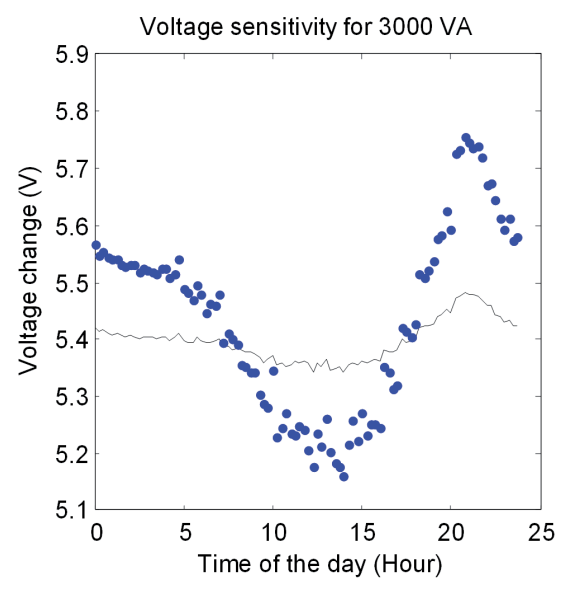

Fig. 6 Voltage sensitivity to $3000 \mathrm{~W}$ injection (dotted) or 3000 var injection (continuous line), straight overhead bare wire line model, end node.

Although the network has close to unity $\mathrm{r} / \mathrm{x}$ ratio (or with overhead cables around 8), the transformer has low $r / x$ ratio. In the case of a single feeder, the voltage drop/rise on the transformer becomes negligible compared to the line after a few sections. However if all of the lines behave in the same way, e.g. all of the loads use or produce more reactive power, it needs further attention. 


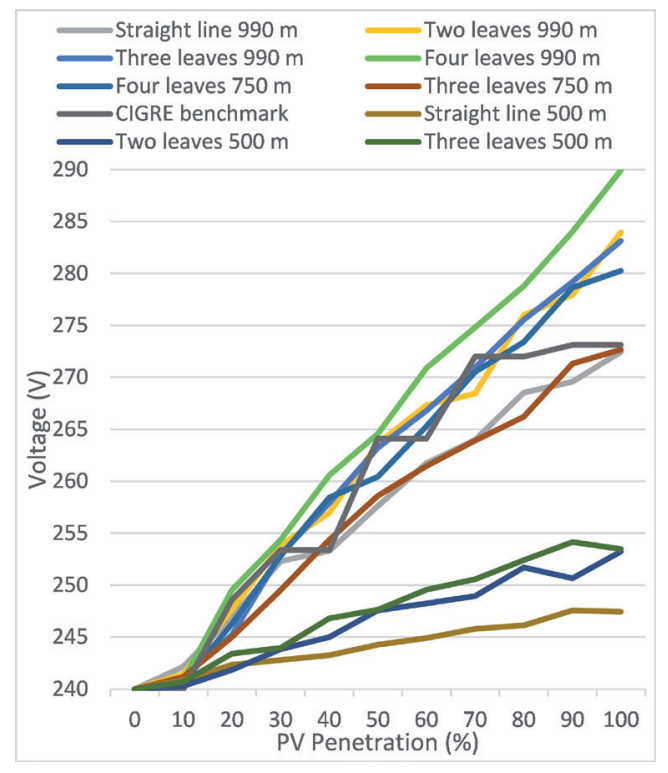

Fig. 7 Highest $90^{\text {th }}$ percentile voltage that was calculated for the networks consisting of overhead lines.

On the other hand, high sensitivity also corresponds to the effective usage of a storage system on the key points of the network. It could help to overcome voltage problems at noon, while also helps to supply customers at night. In the same time, it also helps to mitigate overloading problems.

Extreme cases are unlikely, and do not give general tendencies. Therefore, various scenarios for each model network with PV penetration ranging from $0 \%$ to $100 \%$ were tested. In the case of cable networks, all of the scenarios are viable; the maximum voltage never exceeds $250 \mathrm{~V}$. Hence, only the overhead models are considered in the following.

The nodal voltage with the highest $90^{\text {th }}$ percentile voltage in a day are presented in Fig. 7. As it can be seen, the $90^{\text {th }}$ percentile voltages exceed $250 \mathrm{~V}$ at just around $20 \%$ of PV penetration for the longer lines. If the maximum voltage was considered (Fig. 8), around $10 \%$ can be allowed. The $500 \mathrm{~m}$ variants represent the shorter one third of the overhead network. Even these have voltage problems at greater PV penetrations. The CIGRE benchmark system performs similar to the longer configurations.

The more the maximal voltage the more time of a day cannot be used to produce PV power without smart control. At these times, many of the inverters without any regulation would switch on and off continuously - as the overvoltage protection does its job. So the actual maximal voltage would not exceed $252 \mathrm{~V}$ by large, but the constant switching could cause voltage quality problems.

\section{Applying Simple Regulation}

Two simple regulation strategies are tested. One is the busbar voltage change. This can only be achieved using on load tap changer (OLTC), because during the peak load of nights a higher starting voltage is desirable and a lower one during

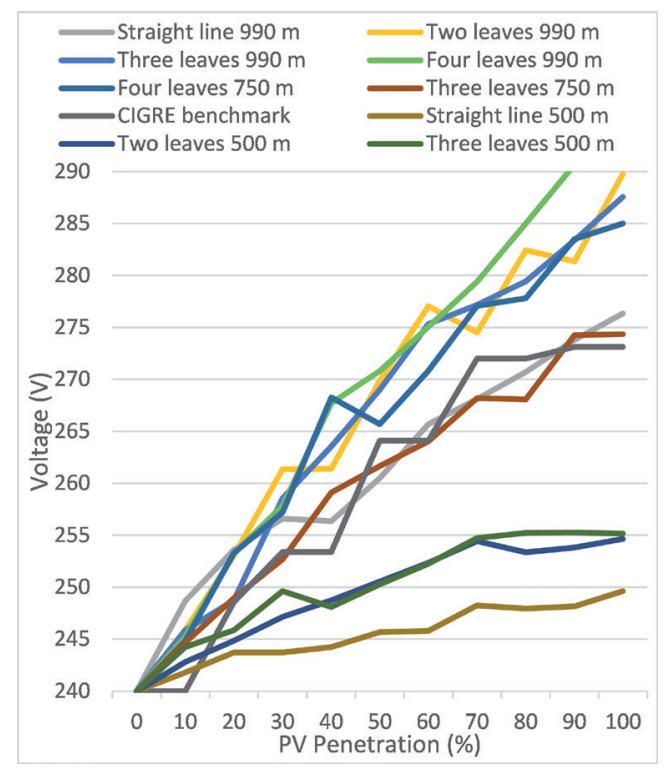

Fig. 8 Maximum voltage that was calculated for the networks consisting of overhead lines.

daytime when the sky is clear. The applicable voltage range is $220-240 \mathrm{~V}$ to avoid under voltage problems. $220 \mathrm{~V}$ was set as busbar voltage during the OLTC tests. In this case (Fig. 9), the allowable PV penetration considerably improved - judging from the highest $90^{\text {th }}$ percentile voltage that appeared during the 50 test runs. It should be noted that this is the case for only one line at a time. If there are multiple feeders connected to a transformer, the busbar voltage has to be set to a value that is acceptable for all of the nodes. This deteriorates its usefulness in cases where some feeders do not have any generation, while the others have much. Still, during daytime a lower voltage could be set in most of the cases.

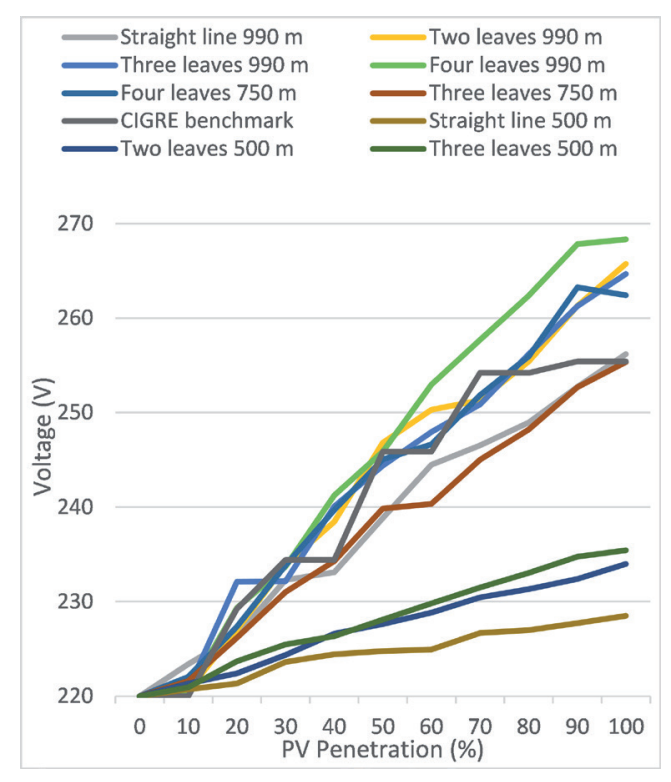

Fig. 9 Highest $90^{\text {th }}$ percentile voltage that was calculated for the overhead lines. Decreased busbar voltage. 
Changing the power injection at nodes with PV generation is another simple method to keep voltages at required levels. Its drawback - in certain cases - is the decreased active power output and increased losses. Also with reactive power injection, a network normally severed by voltage rises can be regulated, but the more the reactive power the more chance it has to overload elements on the network because of the greater magnitude of the current. Moreover, the same problems can happen as with the on load tap changer. That is, if there are some feeders that are heavily feeding back to the grid and while doing so regulate itself with reactive power, they can cause voltage drop on the transformer and so a feeder without generation connected to this transformers could face under voltage problems.

For a simple and safe method only the active power limitation was used with a root voltage of $240 \mathrm{~V}$. The following method was used to decide the active power generation of a household during a 15 minutes interval: The solar irradiation was increased in small increments until the actual irradiation for that specific 15 minutes interval was reached. If the voltage at any point of the network was above the limit in the process, the generator feeding it was curtailed with just the amount required to keep $250 \mathrm{~V}$. This was repeated until no voltage violation occurred and the irradiation reached the value that was used in that 15 minutes interval. This mimics how a real network would behave, therefore customers towards the end of the line are affected more than the ones that are closer to the transformer.

The results indicate that for a portion of energy not produced, the overall generation could be elevated - At noon the average utilization can be low, but this period lasts for only a couple of 15 minutes intervals. The overall average utilization for a feeder line can be seen on Fig. 10.

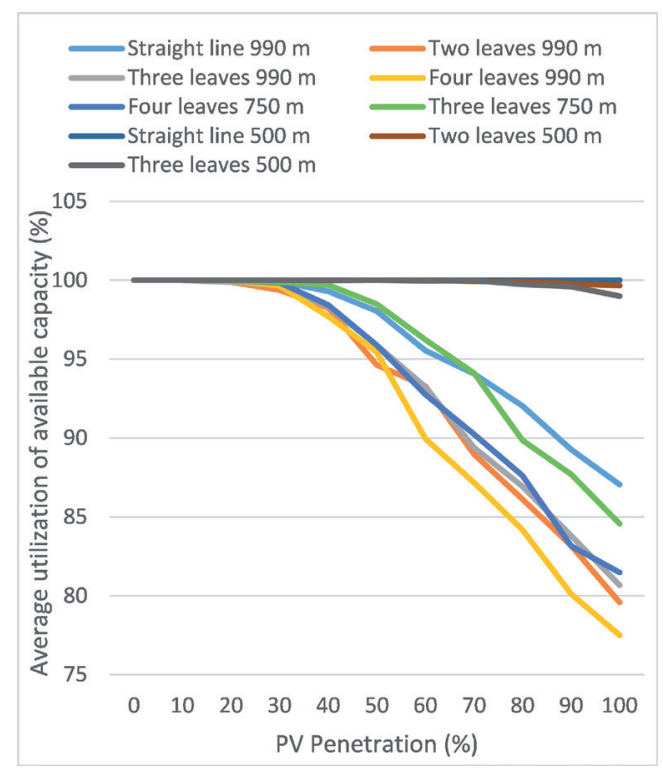

Fig. 10 Average utilization of available capacity during the day, average of 50 test simulations. $-240 \mathrm{~V}$ busbar voltage
It is important to note that this sunny clear test day was the one with the most irradiation in a year. During days with less irradiation, the utilization would be greater. The effect on single customers depends on their distance from the transformer.

It is also worth noting that overloading may be present with high penetration of PV generation.

\section{Conclusion}

A set of sample model networks - including topology, consumption, and generation - were developed, using real, measured data. These can be used to study the behavior of current LV network or can be used as a foundation to build microgrid models.

The current paper analyzed the voltage profiles of these model networks, according to which, a large portion of today's overhead network in Hungary cannot support high levels of PV generation, regardless of the network topology. The studied cases assumed that every household, where there is generation, only produces its own yearly energy consumption. The yearly average household consumption in the sample profiles although matches the country's mean, it is also not particularly large, there are regions where this is much higher $(4000 \mathrm{kWh}$ instead of the $2500 \mathrm{kWh}$ ) and PV penetration is also growing more dynamically there. Expecting growth of yearly consumption also increases the newly installed PV capacity thus decreases the ratio of usable PV generation in the future. Even worse, just a few relatively large PV systems can cause voltage rise problems throughout the network. Moreover, some consumers that plan to install PV system, do it jointly with other investments that significantly increase its consumption - therefore they build a bigger PV capacity (e.g. a larger PV system with heat pump for space heating and cooling). To conclude, it is mandatory to include some voltage regulation techniques in order to accommodate large portion of distributed generation in the future.

The allowable PV penetration in areas where commercial consumers are present would be greater than with residential consumers because the load and production profiles overlap.

Using either or both of the two simple regulations considerably increased the networks' PV potential judged by the voltage profile. However, the high amount of reversed power flow can cause overloading and unexpected results upstream in the MV network that needs further attention.

Typical cable lines should be capable of supporting high PV penetration judging by voltage profiles. Cable and transformer overloading will be the most likely to limit the PV penetration in these areas.

\section{Acknowledgement}

This work was partially supported by the Hungarian Government, financed by National Research, Development and Innovation Fund through project eAutoTech (grant no.: KMR_121-2012-0188). 


\section{References}

[1] Shah, J., Wollenberg, B. F., Mohan, N. "Decentralized Power Flow Control for a Smart Micro-Grid." In: 2011 IEEE Power and Energy Society General Meeting. 24-29 July 2011. DOI: 10.1109/PES.2011.6039396

[2] Laaksonen, H., Saari, P., Komulainen R. "Voltage and frequency control of inverter based weak lv network microgrid." In: Future Power Systems, 2005 International Conference on, Amsterdam, pp. 6. 18-18 Nov. 2005. DOI: $10.1109 /$ fps.2005.204293

[3] Papathanassiou, S., Hatziargyriou, N., Strunz, K. "A Benchmark Low Voltage Microgrid Network." In: CIGRE Symposium Power systems with dispersed generation: Technologies, impacts on development, operation and performances, April 2005.

[4] Kadurek, P., Kling, W. L., Ribeiro, P. F., Cobben, J. F. G. "Electricity demand characterization for analyzing residential LV distribution networks." In: PowerTech (POWERTECH), 2013 IEEE Grenoble, Grenoble, 16-20 June 2013. DOI: 10.1109/PTC.2013.6652168

[5] Giaconia, G. C., Fiscelli, G., Lo Bue, F., Di Stefano, A., La Cascia, D., Miceli, R. "Integration of distributed on site control actions via combined photovoltaic and solar panels system." In: Proceedings of ICCEP 2009, Capri-Italy, pp. 171-177, June 2009. DOI: 10.1109/iccep.2009.5212063

[6] Lampropoulos, I., Vanalme, G. M. A., Kling, W. L. "A methodology for modeling the behavior of electricity prosumers within the smart grid." In: Innovative Smart Grid Technologies Conference Europe (ISGT Europe) 2010 IEEE PES, Gothenburg, 11-13 October 2010.

DOI: 10.1109 /isgteurope.2010.5638967

[7] Canova, A., Giaccone, L., Spertino, F., Tartaglia, M. "Electrical Impact of Photovoltaic Plant in Distributed Network." IEEE Transactions on Industry Applications. 45 (1). pp. 341-347. 2009.

DOI: 10.1109/TIA.2009.2009726
[8] Bollen, M. H. J., Sannino, A. "Voltage Control With Inverter-Based Distributed Generation." IEEE Transactions on Power Delivery. 20 (1). pp. 519-520. 2005. DOI: 10.1109/TPWRD.2004.834679

[9] Shayani, R.A., de Oliveira, M. A. G. "Photovoltaic Generation Penetration Limits in Radial Distribution Systems." IEEE Transactions on Power Systems. 26 (3). pp. 1625-1631. 2011. DOI: 10.1109/TPWRS.2010.2077656

[10] Molina Garcia, A., Mastromauro, R. A., Liserre, M. "A combined centralized/decentralized voltage regulation method for PV inverters in LV distribution networks." In: PES General Meeting 2014, Conference \& Exposition. 2014 IEEE National Harbor, MD, 27-31 July 2014. DOI: 10.1109/PESGM.2014.6938817

[11] Kamperis, D., Vanalme, G. M. A., Kling, W. L. "The ability of a Dutch LV network to incorporate high penetration level of $\mu$-CHPs considering network topology and units control strategy." In: Innovative Smart Grid Technologies (ISGT Europe), 2011 2nd IEEE PES International Conference and Exhibition on. Manchester, 5-7 Dec. 2011.

DOI: 10.1109/isgteurope.2011.6162671

[12] Supponen, A., Repo S. "Impact evaluation of PV generation on LV networks." In: Power Systems Computation Conference (PSCC), 2014. Wroclaw, 18-22 Aug. 2014. DOI: 10.1109/pscc.2014.7038331

[13] Teng, J.-H. "A Direct Approach for Distribution System Load Flow Solutions." IEEE Transactions on Power Delivery. 18 (3). pp. 882-887. 2003. DOI: 10.1109/TPWRD.2003.813818 